\title{
Abnormal phospholipids distribution in the prefrontal cortex from a patient with schizophrenia revealed by matrix-assisted laser desorption/ionization imaging mass spectrometry
}

\author{
Junya Matsumoto $\cdot$ Yuki Sugiura $\cdot$ Dai Yuki $\cdot$ Takahiro Hayasaka $\cdot$ \\ Naoko Goto-Inoue • Nobuhiro Zaima • Yasuto Kunii • Akira Wada • Qiaohui Yang • \\ Keisuke Nishiura • Hiroyasu Akatsu • Akira Hori • Yoshio Hashizume • \\ Takayuki Yamamoto $\cdot$ Keiko Ikemoto $\cdot$ Mitsutoshi Setou $\cdot$ Shin-ichi Niwa
}

Received: 22 November 2010 /Revised: 8 March 2011 /Accepted: 12 March 2011 /Published online: 2 April 2011

(C) The Author(s) 2011. This article is published with open access at Springerlink.com

\begin{abstract}
Schizophrenia is one of the major psychiatric disorders, and lipids have focused on the important roles in this disorder. In fact, lipids related to various functions in the brain. Previous studies have indicated that phospholipids, particularly ones containing polyunsaturated fatty acyl residues, are deficient in postmortem brains from patients with schizophrenia. However, due to the difficulties in handling human postmortem brains, particularly the large size and complex structures of the human brain, there is
\end{abstract}

Published in the special issue Biomedical Mass Spectrometry with Guest Editors Hisao Oka and Mitsutoshi Setou.

Electronic supplementary material The online version of this article (doi:10.1007/s00216-011-4909-3) contains supplementary material, which is available to authorized users.

J. Matsumoto $\cdot$ Y. Kunii $\cdot$ A. Wada $\cdot$ Q. Yang $\cdot$ K. Nishiura $\cdot$

K. Ikemoto $\cdot$ S.-i. Niwa $(\bowtie)$

Department of Neuropsychiatry, School of Medicine,

Fukushima Medical University,

1 Hikarigaoka,

Fukushima, Fukushima 960-1295, Japan

e-mail: si-niwa@fmu.ac.jp

Y. Sugiura $\cdot$ D. Yuki $\cdot$ T. Hayasaka $\cdot$ N. Goto-Inoue $\cdot$ N. Zaima

M. Setou

Department of Molecular Anatomy, Molecular Imaging Frontier

Research Center, Hamamatsu University School of Medicine,

1-20-1, Handayama, Higashi-ku,

Hamamatsu, Shizuoka 431-3192, Japan

H. Akatsu • A. Hori • Y. Hashizume · T. Yamamoto

Choju Medical Institute, Fukushimura Hospital,

Noyori-cho,

Toyohashi, Aichi 441-8124, Japan little agreement regarding the qualitative and quantitative abnormalities of phospholipids in brains from patients with schizophrenia, particularly if corresponding brain regions are not used. In this study, to overcome these problems, we employed matrix-assisted laser desorption/ionization imaging mass spectrometry (IMS), enabling direct microregion analysis of phospholipids in the postmortem brain of a patient with schizophrenia via brain sections prepared on glass slides. With integration of traditional histochemical examination, we could analyze regions of interest in the brain at the micrometric level. We found abnormal phospholipid distributions within internal brain structures, namely, the frontal cortex and occipital cortex. IMS revealed abnormal distributions of phosphatidylcholine molecular species particularly in the cortical layer of frontal cortex region. In addition, the combined use of liquid chromatography/electrospray ionization tandem mass spectrometry strengthened the capability for identification of numerous lipid molecular species. Our results are expected to further elucidate various metabolic processes in the neural system.

Keywords Schizophrenia $\cdot$ Postmortem brain $\cdot$ Imaging mass spectrometry (IMS) · Matrix-assisted laser desorption/ ionization (MALDI) $\cdot$ Phospholipids

\section{Introduction}

Schizophrenia is one of the major psychiatric disorders, producing symptoms such as hallucinations and delusions 
[1]. Despite considerable worldwide research for over a century, the etiology of this illness remains incompletely understood. There are several hypotheses regarding the cause of schizophrenia, and a widely accepted one is the neurotransmitter hypothesis, which involves abnormal synaptic functions due to defects in neurotransmitter regulation, including their production, release, reuptake, and reception by neurotransmitter receptors [2]. Since the neuronal membrane is an important structural and functional site for the receptors, identification of neural lipid abnormalities in postmortem brains of schizophrenia patients has been also intensively pursued.

In the central nervous system, phospholipids are the most abundant lipids. They comprise a molecular family in which phosphoric acid is present in the ester form. Some classes of phospholipids are structurally distinct based on the head group linked to the phosphate attached at the $s n-3$ position of the glycerol backbone, such as phosphatidylcholines (PCs) and phosphatidylinositols (PIs). Depending on the compositions of the two fatty acyl residues linked at the $s n-1$ and $s n-2$ positions, phospholipids of the same class are further subdivided into molecular species. In particular, regarding stored fatty acyl residues in the phospholipids, polyunsaturated fatty acyl residues have been paid special attention in schizophrenia research because they are essential to maintaining normal membrane structure and function as well as to normal brain development [3]. Two types of PUFAs, the $n-6$ and $n-3$ series, primarily arachidonic acid [arachidonate, 20:4(n-6)] and docosahexaenoic acid [DHA, 22:6(n-3)], are designated by the location of the first double bond from the methyl group (see also Electronic Supplementary Material Fig. S1).

So far, the combined use of thin-layer chromatography (TLC) and gas chromatography (GC) has been widely adapted for analyzing lipids in schizophrenia research, which allows the separation of each phospholipid class by TLC, and then quantifying the fatty acid composition of each phospholipid class by GC. In early researches, Horrobin et al. reported defects in the fatty acid composition of phosphatidylethanolamines (PEs), particularly in the frontal cortex of patients with schizophrenia [4]. More recently, Schmitt et al. [5] reported decreased PCs and sphingomyelin (SMs) levels and increased phosphatidylserine (PS) levels in the thalamus. Further, McNamara et al. reported decreased DHA and vaccenic acid levels in the orbitofrontal cortex [6,7]. Mass spectrometry (MS) has been widely adopted in relatively recent schizophrenia research. By using LC-MS, Schwarz et al. reported that PCs and free fatty acids are increased in the frontal cortex of a schizophrenia patient's brain [8].

Despite such intensive studies, however, considerable disagreements exist regarding the qualitative and quantitative abnormalities of lipids in the brains from patients with schizophrenia. For example, Shimon et al. revealed that PI levels are decreased in the postmortem brain of patients [9]; however, Shapiro found no difference in the PI amount by the same method [10]. Hamazaki et al. reported that $n-3$ fatty acids such as DHA were not remarkably altered but that $n-6$ fatty acids, such as arachidonic acid, were decreased in the hippocampus of brains from schizophrenia and bipolar disorder patients [11]. On the other hand, a study focusing on the orbitofrontal area reported a $20 \%$ decrease in DHA in schizophrenia patients [6]. Although several well-recognized reasons lead to variations in the experimental results of postmortem human samples, i.e., ethnogenesis, personal drug and disease history, and postmortem time, the abovementioned cases clearly indicate the importance of controlling experiments by using strictly corresponding brain regions. In fact, current lipid studies, including those by our group, demonstrate that brain lipids are distributed in a heterogeneous pattern even within small brain regions [1214]; therefore, careful and precise dissection techniques are required in such studies, particularly when employing traditional biochemical methods.

Here, we utilized matrix-assisted laser desorption/ionization imaging mass spectrometry (MALDI-IMS) for regional analysis of lipids on brain tissue sections. In this emerging imaging technology, tissue sections were directly scanned by MALDI laser, and mass spectra were recorded for each data point. Based on the ion intensities obtained from each tissue location, two-dimensional distribution maps for multiple lipids could be simultaneously obtained through one measurement [15]. The current spatial resolution of IMS, as defined by laser diameter and accuracy of sample stage movement [16], could examine tissues at micrometric level [17]; we could also directly conduct lipid quantification from microregions on the postmortem brain sections. Furthermore, analyzed tissue sections could be histochemically stained; therefore, the obtained lipid distribution maps could be integrated to traditional histochemical images, thus precisely recognizing the part of brain being analyzed.

In this study, we revealed abnormal PC distributions within internal brain structures, namely, the frontal cortex and occipital cortex of a postmortem brain from a patient with schizophrenia. In addition, the combined use of the LC electrospray ionization tandem mass spectrometry (LC/ESIMS/MS) system strengthened the capability of the technique for molecular identification.

\section{Materials and methods}

Human brain The human brain samples including prefrontal cortex and occipital cortex were obtained following the protocols approved by the Fukushima Medical University. The postmortem brain of a patient with schizophrenia was supplied by the Postmortem Brain Bank of Fukushima for 
Psychiatric Research. This Brain Bank specializes in psychiatric diseases in Japan. The patient with schizophrenia was a 70-year-old male, and the postmortem interval was $18 \mathrm{~h}$. An age- and sex-matched normal sample was obtained from the Choju Medical Institute, Fukushimura Hospital, Toyohashi. The patient characteristics are indicated in Table 1. Informed written consent was obtained from the families of both subjects. The patient with schizophrenia fulfilled the diagnostic criteria established by the American Psychiatric Association (Diagnostic and Statistical Manual of Mental Disorders (DSM-IV)).

Chemicals Methanol, potassium acetate, and ultra pure water were purchased from Wako Chemical (Osaka, Japan). Calibration standard peptide and 2,5-dihydroxybenzoic acid (DHB) were purchased from Bruker Daltonics (Leipzig, Germany). All the chemicals used in this study were of the highest purity available. 9-Aminoacridine was purchased from Acronics (Pittsburgh, PA, USA). 1-Palmitoyl-2-oleylsn-glycero-3-phosphate, PC (diacyl-16/0:18:1), was purchased from Funakoshi Co. Ltd. (Tokyo, Japan).

Tissue section preparation for IMS and ESI-MS/MS Tissues blocks were sectioned at $-18{ }^{\circ} \mathrm{C}$ using a cryostat (CM 1950; Leica, Germany) to a thickness of $8 \mu \mathrm{m}$, as described in previous reports [18, 19]. Although brain blocks were held by an optimum cutting temperature (OCT) polymer, they were not embedded in it because it was considered that any residual polymer on the tissue slices might degrade the mass spectra [19]. The frozen sections were thaw-mounted on indium-tin-oxide (ITO)-coated glass slides (Bruker Daltonics). For LC/ESI-MS/MS, several sections were collected into glass vials for lipid extraction (approximately $10 \mathrm{mg}$ for each), and total lipids were extracted by the Folch method [20].

Sample preparation for LC/ESI-MS/MS The ESI-MS/MS analysis was performed using a 4000Q-TRAP quadrupole linear ion trap hybrid mass spectrometer (Applied Biosystems/ MDS Sciex, Concord, ON, Canada) with an ACQUITY Ultra Performance Liquid Chromatography (LC; Waters, Milford, MA, USA).

A chromatographic method was developed using an ACQUITY UPLCTM BEH C18 column $(2.1 \times 50 \mathrm{~mm}$ i.d.,
1.7- $\mu \mathrm{m}$ particle), fitted with an identically packed guard column $(2.1 \times 5 \mathrm{~mm}$; Waters, Milford, MA, USA). The column oven was maintained at $40^{\circ} \mathrm{C}$. The following gradient elution with mobile phase A (acetonitrile:methanol:water= 19:19:2 $v / v / v$, containing $0.1 \%$ formic acid and $0.028 \%$ ammonia) and mobile phase B (isopropanol, $0.1 \%$ formic acid, and $0.028 \%$ ammonia) was used at a flow rate of $0.4 \mathrm{~mL} / \mathrm{min}: 0-10 \mathrm{~min}: 5 \% \mathrm{~B} \rightarrow 5 \% \mathrm{~B} ; 10-15 \mathrm{~min}: 5 \% \mathrm{~B} \rightarrow$ $50 \% \mathrm{~B} ; 15-20 \min : 50 \% \mathrm{~B} \rightarrow 50 \% \mathrm{~B}$, and $20-25 \min : 5 \% \mathrm{~B}$.

Precursor ion and neutral loss scanning for specific phospholipid classes To identify the specific phospholipid classes, precursor ion scanning of the polar head group of PCs and SMs $(m / z 184)$, and neutral loss scan of that of PE $(\mathrm{m} / \mathrm{z} 141)$ were performed using the 4000Q-TRAP instrument; these methods are effective for detecting PCs because characteristic fragment ions are generated by collisioninduced dissociation (CID) [21]. The level of collision energy is very important to the sensitive identification of focused molecules; therefore, optimal conditions for detecting the appropriate precursor ion and neutral loss were determined by preliminarily analyzing PC (diacyl-16:0/ 18:1) as standard lipid.

Spray coating of the matrix solution for IMS A DHB solution $(40 \mathrm{mg} / \mathrm{mL}$ DHB, $20 \mathrm{mM}$ potassium acetate, $70 \%$ $\mathrm{MetOH}, 0.1 \%$ TFA) was used as the matrix solution for imaging the PCs. Here, 9-aminoacridine $(10 \mathrm{mg} / \mathrm{mL}$, dissolved in $70 \%$ methanol) was used for imaging the PEs. The matrix solution was sprayed over the tissue surface using a 0.2-mm nozzle caliber airbrush (Procon Boy FWA Platinum; Mr. Hobby, Tokyo, Japan). Tissue sections that were to be compared were simultaneously spray-coated with each matrix solution to equalize analyte extraction and co-crystallization conditions. The distance between the nozzle tip and the tissue surface was $10 \mathrm{~cm}$, and the spraying period was fixed at $5 \mathrm{~min}$. Approximately $100 \mu \mathrm{L}$ of matrix solution was sprayed onto each brain section.

IMS conditions (single MS imaging) Single MS imaging was performed using a MALDI TOF/TOF-type instrument (Ultraflex 2 TOF/TOF; Bruker Daltonics). This instrument was equipped with a 355-nm Nd:YAG laser. The data were acquired in the positive reflectron mode under an acceler-
Table 1 Demographic information for subject with schizophrenia and matched control

\begin{tabular}{lll}
\hline & Schizophrenia & Normal control \\
\hline Age at death & 74 & 73 \\
Gender & Male & Male \\
Cause of death & Pneumonia and heart failure & Pneumonia and acute myocardial infarction \\
Brain hemisphere & Left & Left \\
Postmortem interval & $18 \mathrm{~h}$ & $2 \mathrm{~h}$ \\
\hline
\end{tabular}


ating potential of $20 \mathrm{kV}$ using an external calibration method. Signals between $\mathrm{m} / \mathrm{z} 400$ and 1,000 were collected. Raster scans on tissue surfaces were performed automatically using FlexControl and FlexImaging 2.0 software (Bruker Daltonics). The number of laser irradiations was 200 shots in each spot. Image reconstruction was performed using FlexImaging 2.0 software.

$M S / M S$ and $M S / M S$ imaging Molecular identification was performed with LTQ-XL (Thermo Fisher Scientific) equipped with an intermediate-pressure MALDI ion source. Since the ions corresponding to PEs contain multiple ions within the same nominal mass, we performed MS/MS imaging with LTQ-XL at a raster scan pitch of $40 \mu \mathrm{m}$ in negative ion detection mode. Image reconstruction was performed using Image Quest (Thermo Fisher Scientific).

Data analysis of IMS We extracted the spectra in about 200 data point from gray matter by FlexImaging 2.0 software (Bruker Daltonics), and the intensities of assigned mass peaks were quantitated by FlexAnalysis 3.0 software (Bruker Daltonics). To relatively quantitative analyses, the mean intensity ratio of schizophrenia/normal of each assigned peaks was calculated in the frontal cortex and occipital cortex, and $p$ value was calculated by $t$ test using relative intensities of each data point in gray matter region to compare mean intensity ratio between schizophrenia and normal brain. Statistical differences between the data from the patient with schizophrenia and the control subject were assessed using an unpaired Student's $t$ test. $p<0.01$ was considered to be significant.

\section{Results and discussion}

\section{Experimental scheme}

Figure 1 shows the experimental scheme employed in this study. In hospitals, including brain bank-network institutes, because of the large size and complex structure of human brains, dissected postmortem brains are generally sectioned into several blocks and then stored at $-80{ }^{\circ} \mathrm{C}$ until further use. In this study, we focused upon the frontal and occipital parts of the cerebral cortex, particularly because the frontal cortex region is known to exhibit apparent anatomical defects in the brains of schizophrenia patients [22, 23]. Frozen brain blocks were thinly sliced, and for LC/ESI-MS/ MS, several sections were collected into glass vials for lipid extraction. Further, few successive sections were thawmounted on the ITO-coated glass slides for the MALDI imaging experiment. Total lipids were extracted from the collected tissues in the vials, and molecular species

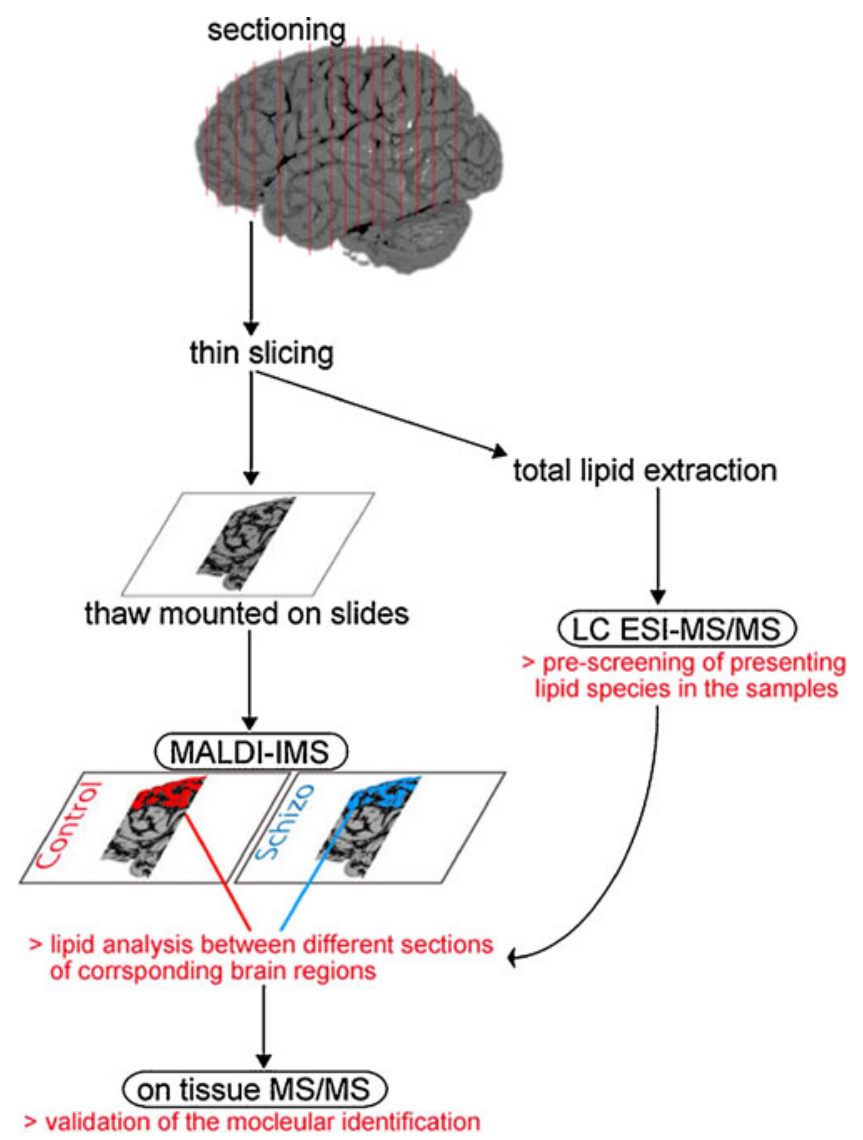

Fig. 1 Scheme of experimental procedure employing MALDI-IMS and LC/ESI-MS/MS for microregion lipid analysis of human postmortem brain. Dissected postmortem brains were sectioned into several blocks and then stored at $-80{ }^{\circ} \mathrm{C}$ until use. Frozen brain blocks were thinly sliced in the cryo-chamber, and for LC/ESI-MS/MS, several sections were collected into glass vials for lipid extraction. Successive sections were then thaw-mounted on the ITO-coated glass slides for the MALDI imaging experiment. Lipids were extracted, and the molecular species composition of specific phospholipid classes was selectively identified by LC/ESI-MS/MS. Based on the identified molecular species composition, lipid species whose intensities changed between the control and diseased brains were screened from the mass spectra obtained from the specified brain region in the imaging data. Finally, the extracted candidates were confirmed as a designated lipid species by MS/MS on the tissue section

compositions of specific phospholipid classes were selectively identified by MS/MS scans, provided by LC/ESIMS/MS. Next, MALDI-IMS was performed on the successive tissue sections, and ion peaks corresponding to the preidentified lipid molecular species by LC/ESI-MS/MS were selected. Since mass spectra obtained by imaging have spatial information, in the next step, we analyzed only spectra from corresponding brain regions of the normal control and patient, although the shapes of the dissected brain blocks differed considerably. Using mass spectra extracted only from the frontal and occipital cortex regions, we identified lipid species whose detected ion intensities changed between the control and diseased brains. Finally, 
for the identified lipid species with abnormal expression amounts in the patient's brain, we validated them as designated lipid species by MS/MS of the tissue section.

Identification of individual molecular species of PCs, SMs, and PEs by LC/ESI-MS/MS

Before conducting MALDI-IMS experiments, we selectively identified the composition of lipid molecular species, namely, PCs, SMs, and diacyl-PEs in the total lipid extract from normal human brain using LC/ESI-MS/MS. By precursor ion scanning of the choline containing head group, we preidentified PC and SM molecular species; diacyl-PE molecular species were identified by neutral loss scan of the phosphoethanolamine containing head group (Fig. 2). Such preelucidation of the targeted phospholipid molecular species was helpful in the IMS data interpretation described later. Since IMS experiments directly subject tissue section samples that are complex mixtures of numerous biological molecules, mass spectra obtained by IMS comprised hundreds of peaks derived from biological molecules as well as matrix clusters. In MALDI-IMS, molecular identification of such ions one by one is a time-consuming process. In this study, by pre-identifying the existing lipid molecular species by LC/ESI-MS/MS, we could pre-define the target mass peaks in the following IMS data analysis. This would be particularly important for a study with samples from human patients because available sample amounts are severely limited.

For the identification of phospholipids containing the choline head group, such as PC, lysoPC, and SM species, a specific fragment at $m / z 184$ (phosphorylcholine) is known and has been used as a specific target in identifying choline-containing phospholipids using product ion and precursor ion scanning methods [24-27]. Figure 2a shows a two-dimensional (2D) abundance map of PC and SM molecular species from the human brain obtained by $\mathrm{LC} /$ ESI-MS using the $\mathrm{C} 18$ reverse-phase column in the positive ion detection mode. The 2D map has the $\mathrm{m} / \mathrm{z}$ value along the vertical axis and the retention time along the horizontal axis. The total ion chromatogram and averaged mass spectra are shown on the upper and left sides of the 2D map, respectively. When using a reverse-phase column, PCs and SMs elute in order from the hydrophilic to the more hydrophobic species. The length of fatty acyl chains mainly influences the elution order, and the number of double bonds in fatty acyl chains also influences the elution order. Based on the $\mathrm{m} / \mathrm{z}$ value and relative retention time and previous reports $[28,29]$, we assigned 17 PC molecular species and three SM species as abundant PC and SM molecules in the human brain (Fig. 2b). In the same way, we also identified 11 diacyl-PE species by neutral loss scanning of $\mathrm{m} / \mathrm{z} 141$ (phosphoethanolamine head group), also in positive ion detection mode (Fig. 2c, d). One thing we have to note is that plasmalogen-type PE were less detected by this ESI-MS/MS method (J Am Soc Mass Spectrom 15(10):[36]). Having obtained qualitative data regarding molecular identification, we next proceeded to MALDI-IMS of the normal control and schizophrenia brains.

Microregion analysis of phospholipid molecular species in the brain sections

Next, we proceeded to microregion analysis of lipids in the brain sections from the control subject and the patient with schizophrenia. First, we performed MALDI-IMS of both brain sections in the positive ion detection mode. We employed an optimized experimental condition for PC and SM detection including composition of matrix solution [14] in order to selectively analyze PC and SM species. In particular, potassium salts added to the matrix solution caused merging of various lipid adducts (adducts with proton, sodium, and potassium) into one single potassiated species, thus making IMS data interpretation fairly easy [30]. After the IMS measurement, applied matrix crystals were removed by immersing the slides into methanol, and the tissue sections were stained with hematoxylin and eosin (HE). Stained sections were then observed by light microscopy, and based on the obtained microscopic image, we defined the cerebral cortex region of both sections (Fig. 3a) and applied them to the IMS data set.

Because of the low availability of postmortem human brain, it is generally difficult to acquire tissue blocks containing complete corresponding brain regions. In this case, the control brain block contained only gray matter regions, while the brain block from the schizophrenia patient contained both gray and white matter. We extracted data only from the corresponding brain areas by the imaging-based analysis. We defined regions of interest (ROI) on the frontal and occipital lobes, according to the microscopic images of HE-stained tissue sections. From the defined regions of interest, approximately 200 spectra for each were collected, and mass peaks were detected and then their intensities were calculated. Electronic Supplementary Material Fig. S2 shows the representative mass spectrum obtained; from the numerous mass peaks detected, we assigned 17 mass peaks as the pre-identified PC and SM molecular species. Ion intensity values of the 17 ions from the extracted spectra ( 800 spectra in total) were calculated and compared between the control and patient samples.

The results are summarized in Table 2. In the table, the green cells represent increase in the amount, while red represents a reduced amount in the patient as compared to the normal control. Table lines representing each lipid species are arranged in order of the greatest increasing to 
Fig. 2 Identification of individual molecular species of focused phospholipid classes by precursor ion and neutral loss scanning of their head groups in the positive ion mode. The extracted total lipid mixture from the control postmortem human brain was subjected to precursor ion scanning of $m / z 184$ for detection of PCs and SMs $(\mathbf{a}, \mathbf{b})$ and neutral loss scan of $m / z 141$ for detection of diacyl form PEs (c, d). The 2D map (a, c) has the $m /$ $z$ value along the vertical axis and the retention time along the horizontal axis. The total ion chromatogram and averaged mass spectra were shown on the upper and left sides of the 2D map, respectively. From the 2D map, the relative ion counts of each PC, SM, and PE molecule species were calculated (The most intense species is shown as intensity at 1.) Detected PUFAs, namely, arachidonic acid, DHA, and docosatetraenoic acid, are shown as red, orange, and green characters, respectively

\section{(a) Precursor ion scan of $m / z 184$}

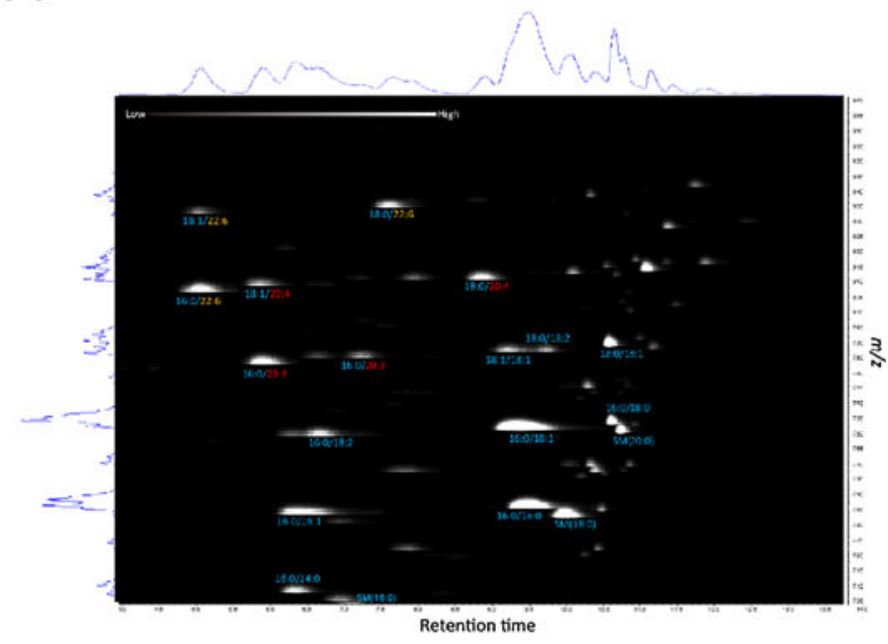

(b)

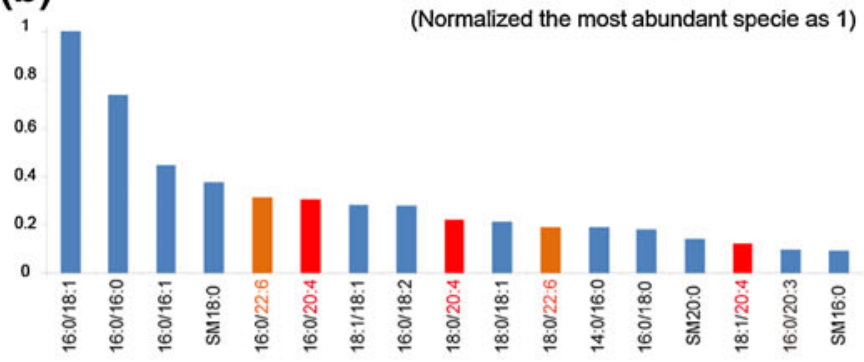

(c)

Neutral loss scan of $m / z 141$

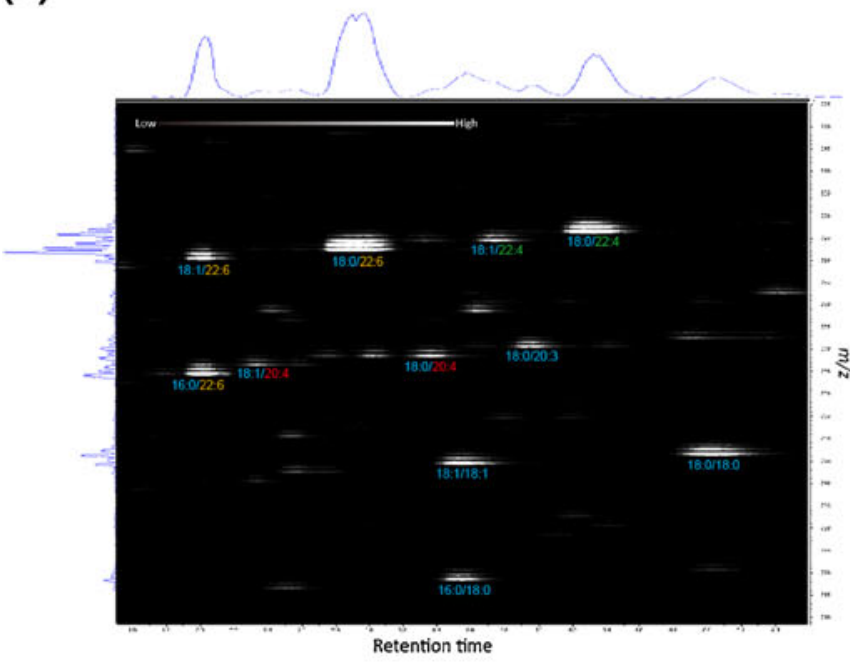

(d)

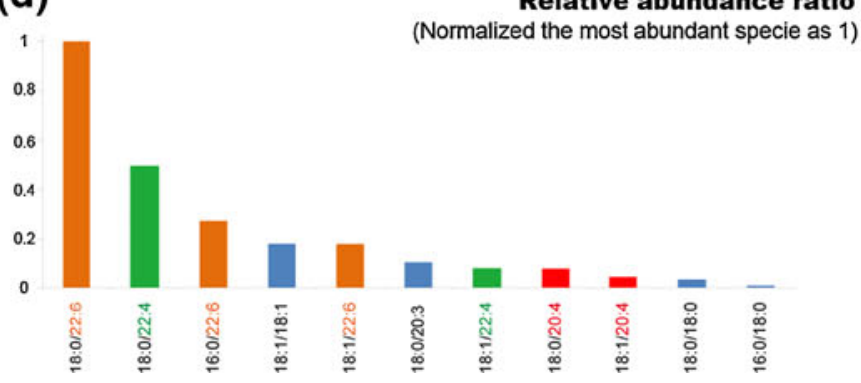




\section{A H-E stained}

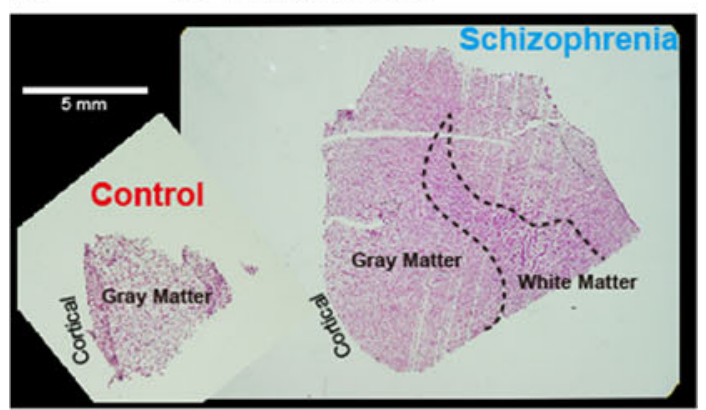

B

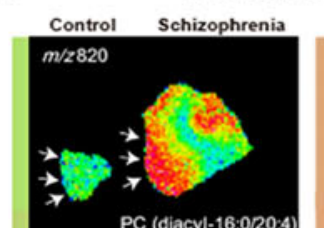

PC (diacyl-16:0/20:4)
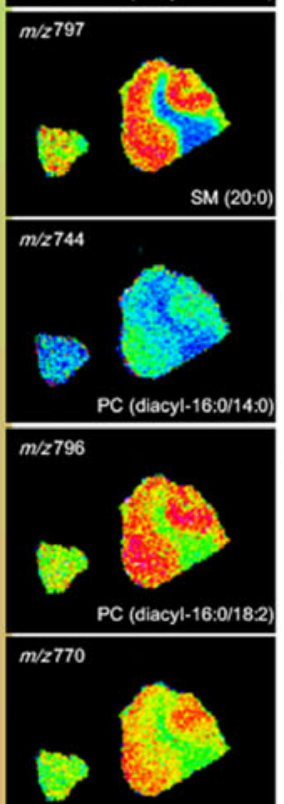

PC (diacyl-16:0/16:1)
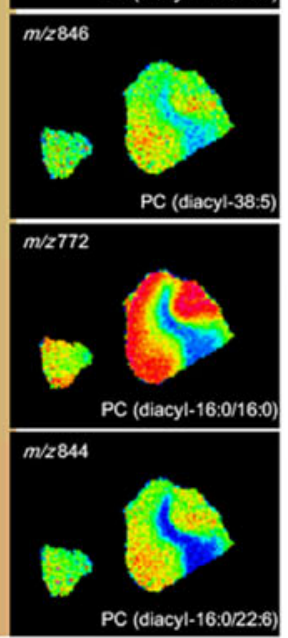

PC (diacyl-16:0/22:

high

Fig. 3 Visualization of PC distributions showing changes in the detection intensities between the brains of control and the patient with schizophrenia. a Microscopic observation of HE-stained brain sections analyzed after IMS measurement. b IMS results for brain sections from control and the patient with schizophrenia

decreasing trend in the frontal cortex of the patient. PC (diacyl-16:0/20:4) was the most increased species in the frontal cortex, while PC (diacyl-16:0/18:1) was the most reduced species in both the frontal and occipital cortex. Here, we particularly focused on the molecular species whose level was significantly altered in the frontal but not in the occipital cortex because such an abnormal pattern may reflect anatomical defects that may be attributed to schizophrenia [22, 23]; this molecule was identified as PC (diacyl-16:0/20:4).

In Fig. 3b, we have shown the distribution of the PCs. Ion images are arranged in the same order as in Table 2. These images demonstrated that each PC and SM molecular species have a rather heterogeneous distribution pattern even within cerebral cortex brain blocks, implicating the importance of precise tissue dissection when employing conventional biochemical analysis. On the other hand, taking advantage of the imaging analysis, we could adjust accurate corresponding regions for comparing differently shaped samples. In Fig. 4a, b, we plotted ion abundances of the PCs along with the distance from the cortical to the deep layer of the cerebral cortex. The graphs for PC (diacyl-16:0/ 20:4) and PC (diacyl-16:0/18:1) revealed that both-the increase in PC (diacyl-16:0/20:4) and the reduction in PC (diacyl-16:0/18:1) - in the patient were particularly observed in the cortical layer of the cortex (arrows). Regarding the PEs, since their ionization efficiency was relatively low in the positive ion detection mode when compared to the choline-containing lipids, we analyzed these in the negative detection mode using 9-aminoaciridine as a matrix [31]. Furthermore, to achieve a high detection selectivity, we performed MS/MS imaging of the PE species for two abundant pre-identified species because PCs remain detectable as $-\mathrm{CH}^{+}$ions if 9-aminoaciridine is used as a matrix in the negative ion detection mode [32]. In fact, as Electronic Supplementary Material Fig. S3 shows, ions corresponding to PEs contained several ions other than PE, but we could successfully visualize PE (diacyl-18:0/22:6) and PE (diacyl18:0/22:4) by visualizing ion transition in which specific fatty acyl residues were produced from their precursor ions (Fig. 4c and Electronic Supplementary Material Fig. S3), though significant alteration was not altered.

Finally, we validated the assignments of PC molecular species by tissue MS/MS, particularly for molecules demonstrating considerable alteration rates in Table 2 . Figure 5 shows the product ion spectra for the ions corresponding to the six PC and SM molecules; in each spectrum, the presence of a choline containing head group 
Table 2 Molecular species of PCs and SMs in order of the increased rate in the frontal cortex from the patient with schizophrenia as compared to the normal control, as revealed by MALDI-IMS

\begin{tabular}{|c|c|c|c|c|c|}
\hline \multirow[t]{2}{*}{ Molecular weight } & \multirow[t]{2}{*}{ LC/ESI-MS/MS } & \multicolumn{2}{|c|}{$\begin{array}{l}\text { Rate of increase in frontal cortex compared to } \\
\text { normal }\end{array}$} & \multicolumn{2}{|c|}{$\begin{array}{l}\text { Rate of increase in occipital cortex compared to } \\
\text { normal }\end{array}$} \\
\hline & & Ratio & $p$ value & Ratio & $p$ value \\
\hline 820.53 & PC (diacyl-16:0/20:4) & 1.32 & $<0.01$ & 1.09 & $<0.01$ \\
\hline 797.59 & SM (20:0) & 1.26 & $<0.01$ & 1.30 & $<0.01$ \\
\hline 744.49 & PC (diacyl-16:0/14:0) & 1.18 & $<0.01$ & 1.13 & $<0.01$ \\
\hline 796.53 & PC (diacyl-16:0/18:2) & 1.12 & $<0.01$ & 1.02 & 0.21 \\
\hline 770.51 & PC (diacyl-16:0/16:1) & 1.10 & $<0.01$ & 0.95 & 0.01 \\
\hline 846.54 & PC (diacyl-18:1/20:4) & 1.08 & $<0.01$ & 1.08 & $<0.01$ \\
\hline 772.53 & PC (diacyl-16:0/16:0) & 1.07 & 0.01 & 0.84 & $<0.01$ \\
\hline 844.53 & PC (diacyl-16:0/22:6) & 1.03 & 0.14 & 1.04 & 0.04 \\
\hline 870.54 & PC (diacyl-18:1/22:6) & 1.00 & 0.95 & 1.27 & $<0.01$ \\
\hline 848.56 & PC (diacyl-18:0/20:4) & 0.99 & 0.57 & 0.77 & $<0.01$ \\
\hline 826.57 & PC (diacyl-18:0/18:1) & 0.98 & 0.39 & 0.93 & $<0.01$ \\
\hline 769.56 & SM (18:0) & 0.98 & 0.30 & 0.82 & $<0.01$ \\
\hline 872.56 & PC (diacyl-18:0/22:6) & 0.97 & 0.04 & 1.13 & $<0.01$ \\
\hline 800.56 & PC (diacyl-16:0/18:0) & 0.97 & 0.06 & 0.86 & $<0.01$ \\
\hline 824.56 & PC (diacyl-18:1/18:1) & 0.94 & $<0.01$ & 0.85 & $<0.01$ \\
\hline 822.54 & PC (diacyl-16:0/20:3) & 0.94 & $<0.01$ & 0.94 & $<0.01$ \\
\hline 798.54 & PC (diacyl-16:0/18:1) & 0.86 & $<0.01$ & 0.75 & $<0.01$ \\
\hline
\end{tabular}

These molecular species were identified by LC/ESI-MS/MS

and phosphate was confirmed. Neutral losses (NL) of 59 and $183 \mathrm{u}$ from precursor ions, corresponding to trimethylamine and phosphocholine, respectively, were used as diagnostic ions in product ion mass spectra [33]. Furthermore, we confirmed detailed fatty acid constituents of each PC by replacing adducted alkali metal from potassium to lithium, by adding $20 \mathrm{mM}$ of lithium acetate in the matrix solution [30]. MS/MS subjecting such lithium-adducted molecules provides increased acyl-chain loosed fragment ions; therefore, we could precisely identify the fatty acid moiety ([NL] of acyl-chain; see Electronic Supplementary Material

Fig. 4 Regional analysis of PC and PE molecular species in the cerebral cortex of postmortem human brains. In-depth analyses of the most increased PCs (16:0/ 20:4) (a) and the most decreased one (16:0/18:1) (b), and PE (18:0/22:6) (c). Plots of ion abundances of the PCs and PE along with the distance from the cortical to the deep layer of the cerebral cortex, demonstrating that the cortical layer showed maximal changes in the lipid contents (arrows)
A
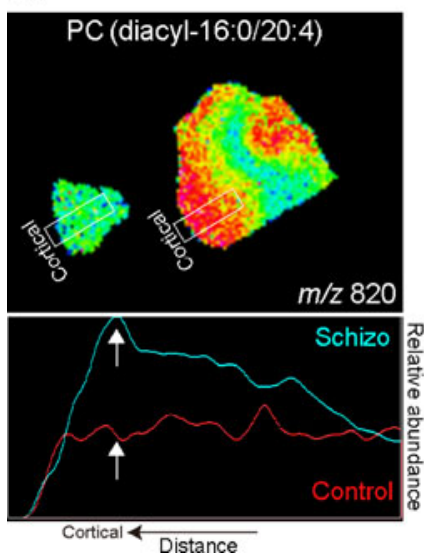

Figs. S4 and S5). Overall, the six molecular species were identified as PC and SM molecules. On the other hand, the ion at $m / z 848$ was revealed to contain both PC (diacyl-18:0/ 20:4) and galactosylceramide, and therefore, its data was eliminated from this investigation (data not shown). We also note regarding lyso-phospholipids; we certainly detected the lyso-phospholipid species particularly of the lyso-PCs (LPCs) by both MALDI and ESI-MS. However, since their amounts are much smaller than diacyl-phospholipids, therefore, they are likely to be sensitive to postmortem degradation. Thus, we here omitted discussion on them.
B
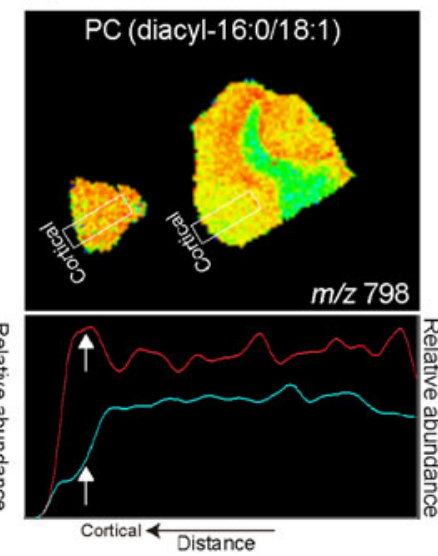

C
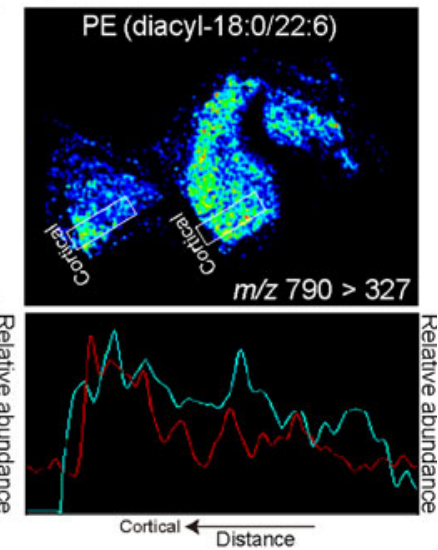
increased ions

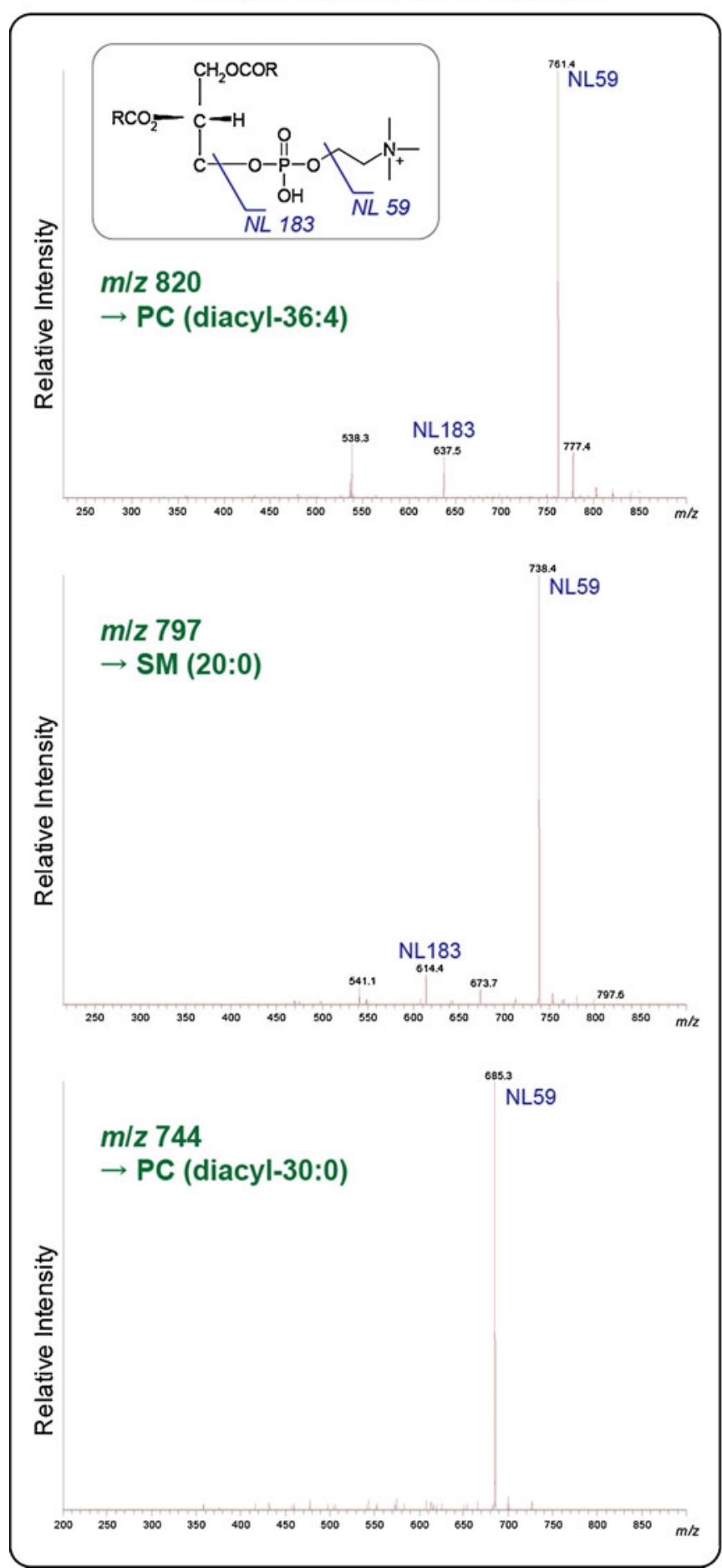

Fig. 5 On tissue MS/MS of molecules showing considerably changed ratios. In each mass spectrum, the presence of a choline containing head group and phosphate was confirmed (neutral losses [NL] of 59

Lipid analysis linked to brain functional mapping in postmortem human brains

Through this study, we found that PC (diacyl-16:0/20:4) containing arachidonic acid was increased in the prefrontal decreased ions

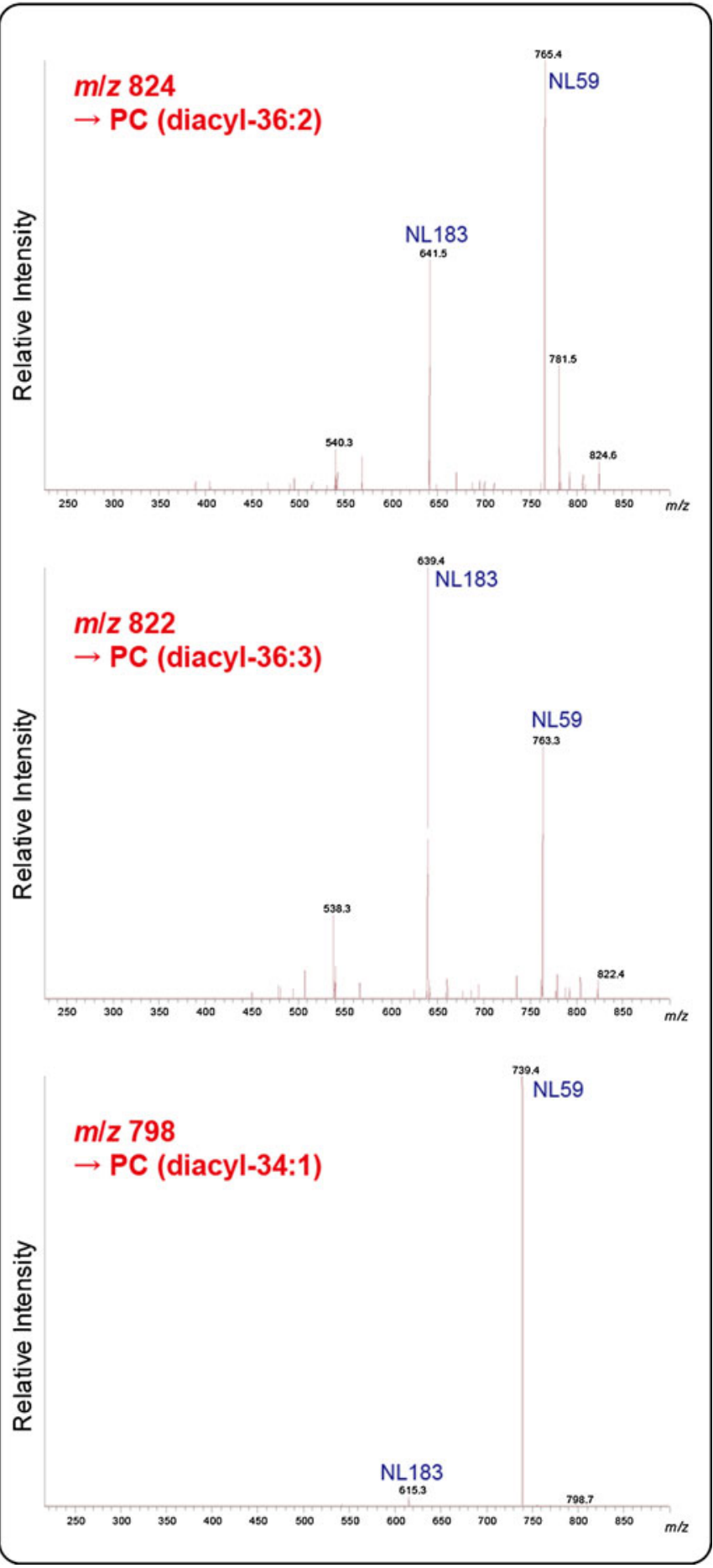

and $183 \mathrm{u}$ from precursor ions, corresponding to trimethylamine and trimethylamine with cyclophosphate, respectively); these were used as the diagnostic ions in product ion mass spectra [33]

cortex of the patient with schizophrenia; no such increase was found in the occipital cortex. As mentioned above, we particularly focused on the molecular species whose level showed a significant alteration in the frontal but not in the occipital cortex because such a defect pattern may reflect 
the anatomical defects attributable to schizophrenia [22, 23]. Our finding agrees with those of a previous study by McNamara et al. who found that among male schizophrenia patients, the arachidonic acid:DHA ratio was increased in the frontal cortex [6].

Our methodology in this study had the specific advantage of enabling small-region analysis of lipid molecular species along with spatial information. This could uncover abnormalities in local lipid metabolism within postmortem human brains. Each region of the human brain cortex is known to have heterogeneous functions, especially highlevel ones, including social skills for communication [34]. Since schizophrenia patients exhibit abnormalities in such higher function, it is particularly important to link previous biochemical analyses - including lipid analyses - to brain functional mapping. We propose that our methodology would enable such evaluation. Although the present study is only a feasibility study, we are currently conducting large-scale case-control studies using postmortem brains provided by our brain bank network [35].

Open Access This article is distributed under the terms of the Creative Commons Attribution Noncommercial License which permits any noncommercial use, distribution, and reproduction in any medium, provided the original author(s) and source are credited.

\section{References}

1. van Os J, Kapur S (2009) Schizophrenia. Lancet 374(9690):635645

2. Howes OD, Kapur S (2009) The dopamine hypothesis of schizophrenia: version III- the final common pathway. Schizophr Bull 35(3):549-562. doi:10.1093/schbul/sbp006

3. Tacconi MT, Calzi F, Salmona M (1997) Brain lipids and diet. In: Hillbrand M, Spitz TS (eds) Lipids, health and behavior. American Psychological Association, Washington, pp 197-226

4. Horrobin DF, Manku MS, Hillman H, Iain A, Glen M (1991) Fatty acid levels in the brains of schizophrenics and normal controls. Biol Psychiatry 30(8):795-805

5. Schmitt A, Wilczek K, Blennow K, Maras A, Jatzko A, Petroianu G, Braus DF, Gattaz WF (2004) Altered thalamic membrane phospholipids in schizophrenia: a postmortem study. Biol Psychiatry 56(1):41-45

6. McNamara RK, Jandacek R, Rider T, Tso P, Hahn CG, Richtand NM, Stanford KE (2007) Abnormalities in the fatty acid composition of the postmortem orbitofrontal cortex of schizophrenic patients: gender differences and partial normalization with antipsychotic medications. Schizophr Res 91(1-3):37-50

7. McNamara RK, Hahn CG, Jandacek R, Rider T, Tso P, Stanford KE, Richtand NM (2007) Selective deficits in the omega-3 fatty acid docosahexaenoic acid in the postmortem orbitofrontal cortex of patients with major depressive disorder. Biol Psychiatry 62 (1): $17-24$

8. Schwarz E, Prabakaran S, Whitfield P, Major H, Leweke FM, Koethe D, McKenna P, Bahn S (2008) High throughput lipidomic profiling of schizophrenia and bipolar disorder brain tissue reveals alterations of free fatty acids, phosphatidylcholines, and ceramides. J Proteome Res 7(10):4266-4277
9. Shimon H, Sobolev Y, Davidson M, Haroutunian V, Belmaker RH, Agam G (1998) Inositol levels are decreased in postmortem brain of schizophrenic patients. Biol Psychiatry 44(6):428-432

10. Shapiro J, Belmaker RH, Biegon A, Seker A, Agam G (2000) Scyllo-inositol in post-mortem brain of bipolar, unipolar and schizophrenic patients. J Neural Transm 107(5):603-607

11. Hamazaki K, Choi KH, Kim HY (2010) Phospholipid profile in the postmortem hippocampus of patients with schizophrenia and bipolar disorder: no changes in docosahexaenoic acid species. J Psychiatr Res 44(11):688-693

12. Murphy RC, Hankin JA, Barkley RM (2009) Imaging of lipid species by MALDI mass spectrometry. J Lipid Res 50(Suppl): S317-S322

13. Garrett TJ, Prieto-Conaway MC, Kovtoun V, Bui H, Izgarian N, Stafford G, Yost RA (2006) Imaging of small molecules in tissue sections with a new intermediate-pressure MALDI linear ion trap mass spectrometer. Int J Mass Spectrom 260:11

14. Sugiura Y, Konishi Y, Zaima N, Kajihara S, Nakanishi H, Taguchi R, Setou M (2009) Visualization of the cell-selective distribution of PUFA-containing phosphatidylcholines in mouse brain by imaging mass spectrometry. J Lipid Res 50(9):1776-1788

15. Sugiura Y, Setou M (2010) Imaging mass spectrometry for visualization of drug and endogenous metabolite distribution: toward in situ pharmacometabolomes. J Neuroimmune Pharmacol 5(1):31-43

16. Chaurand P, Schriver KE, Caprioli RM (2007) Instrument design and characterization for high resolution MALDI-MS imaging of tissue sections. J Mass Spectrom 42(4):476-489

17. Harada T, Yuba-Kubo A, Sugiura Y, Zaima N, Hayasaka T, GotoInoue N, Wakui M, Suematsu M, Takeshita K, Ogawa K, Yoshida Y, Setou M (2009) Visualization of volatile substances in different organelles with an atmospheric-pressure mass microscope. Anal Chem 81(21):9153-9157

18. Sugiura Y, Shimma S, Setou M (2006) Thin sectioning improves the peak intensity and signal-to-noise ratio in direct tissue mass spectrometry. J Mass Spectrom Soc Jpn 54(2):4

19. Schwartz SA, Reyzer ML, Caprioli RM (2003) Direct tissue analysis using matrix-assisted laser desorption/ionization mass spectrometry: practical aspects of sample preparation. J Mass Spectrom 38(7):699-708

20. Folch J, Lees M, Sloane Stanley GH (1957) A simple method for the isolation and purification of total lipides from animal tissues. $\mathrm{J}$ Biol Chem 226(1):497-509

21. Taguchi R, Houjou $T$, Nakanishi H, Yamazaki T, Ishida $M$, Imagawa M, Shimizu T (2005) Focused lipidomics by tandem mass spectrometry. J Chromatogr B Analyt Technol Biomed Life Sci 823(1):26-36

22. McCarley RW, Wible CG, Frumin M, Hirayasu Y, Levitt JJ, Fischer IA, Shenton ME (1999) MRI anatomy of schizophrenia. Biol Psychiatry 45(9):1099-1119

23. Sun D, Stuart GW, Jenkinson M, Wood SJ, McGorry PD, Velakoulis D, van Erp TG, Thompson PM, Toga AW, Smith DJ, Cannon TD, Pantelis C (2009) Brain surface contraction mapped in first-episode schizophrenia: a longitudinal magnetic resonance imaging study. Mol Psychiatry 14(10):976-986. doi:10.1038/ mp.2008.34

24. Houjou T, Yamatani K, Nakanishi H, Imagawa M, Shimizu T, Taguchi R (2004) Rapid and selective identification of molecular species in phosphatidylcholine and sphingomyelin by conditional neutral loss scanning and MS3. Rapid Commun Mass Spectrom 18(24):3123-3130

25. Smith PB, Snyder AP, Harden CS (1995) Characterization of bacterial phospholipids by electrospray ionization tandem mass spectrometry. Anal Chem 67(11):1824-1830

26. Ekroos K, Chernushevich IV, Simons K, Shevchenko A (2002) Quantitative profiling of phospholipids by multiple precursor ion 
scanning on a hybrid quadrupole time-of-flight mass spectrometer. Anal Chem 74(5):941-949

27. Cole MJ, Enke CG (1991) Direct determination of phospholipid structures in microorganisms by fast atom bombardment triple quadrupole mass spectrometry. Anal Chem 63(10):1032-1038

28. Houjou T, Yamatani K, Imagawa M, Shimizu T, Taguchi R (2005) A shotgun tandem mass spectrometric analysis of phospholipids with normal-phase and/or reverse-phase liquid chromatography/ electrospray ionization mass spectrometry. Rapid Commun Mass Spectrom 19(5):654-666

29. Nakanishi H, Iida Y, Shimizu T, Taguchi R (2010) Separation and quantification of sn-1 and sn-2 fatty acid positional isomers in phosphatidylcholine by RPLC-ESIMS/MS. J Biochem 147(2):245-256

30. Sugiura Y, Setou M (2009) Selective imaging of positively charged polar and nonpolar lipids by optimizing matrix solution composition. Rapid Commun Mass Spectrom 23(20):3269-3278

31. Touboul D, Brunelle A, Laprevote O (2011) Mass spectrometry imaging: towards a lipid microscope? Biochimie 93(1):113-119
32. Fuchs B, Bischoff A, Suss R, Teuber K, Schurenberg M, Suckau D, Schiller J (2009) Phosphatidylcholines and -ethanolamines can be easily mistaken in phospholipid mixtures: a negative ion MALDI-TOF MS study with 9-aminoacridine as matrix and egg yolk as selected example. Anal Bioanal Chem 395(8):2479-2487

33. Stubiger G, Pittenauer E, Allmaier G (2008) MALDI seamless postsource decay fragment ion analysis of sodiated and lithiated phospholipids. Anal Chem 80(5):1664-1678

34. Pinkham AE, Penn DL (2006) Neurocognitive and social cognitive predictors of interpersonal skill in schizophrenia. Psychiatry Res 143(2-3):167-178

35. Ikemoto K, Niwa S (2007) Proceedings of the 1st Symposium for Brain Bank, 22 October 2006, Fukushima, Japan. Psychiatry Clin Neurosci 61:S19-S23. doi:10.1111/j.1440-1819.2007.01647.x

36. Zemski Berry KA, Murphy RC (2004) Electrospray ionization tandem mass spectrometry of glycerophosphoethanolamine plasmalogen phospholipids. J Am Soc Mass Spectrom 15(10):14991508 